Original Research Article

\title{
A prospective open labelled study of effect of vildagliptin with metformin in patients with type 2 diabetes mellitus
}

\author{
Lavakumar S. ${ }^{1}$, Jacob Jesurun R. S. ${ }^{2 *}$
}

${ }^{1}$ Department of Pharmacology, Shri Sathya Sai Medical College and Research Institute, SBV deemed to be university, Kanchipuram, Puducherry, India ${ }^{2}$ Department of Pharmacology, Believers Church Medical College And Hospital, Thiruvalla, Kerala, India

Received: 02 June 2019

Revised: 30 June 2019

Accepted: 06 July 2019

\section{*Correspondence to:}

Dr. Jacob Jesurun R.S, Email: sathishkumard11@ gmail.com

Copyright: (C) the author(s), publisher and licensee Medip Academy. This is an openaccess article distributed under the terms of the Creative Commons Attribution NonCommercial License, which permits unrestricted noncommercial use, distribution, and reproduction in any medium, provided the original work is properly cited.

\begin{abstract}
Background: The pathophysiology of Type 2 Diabetes Mellitus (T2DM) is characterized by deficient insulin activity arising from decreased insulin secretion secondary to beta cell failure, and/or compromised insulin action in peripheral target tissues (insulin resistance).

Methods: The patients attending the medicine outpatient department of tertiary care teaching hospital were enrolled in the study. Patients, who fulfilled the selection criteria, were allocated in two treatment groups. Group A was treated with metformin (Sustained release preparation) $500 \mathrm{mg}$ once daily and group B was treated with vildagliptin $50 \mathrm{mg}$ once daily. Measurement of body weight, fasting blood glucose (FPG), postprandial blood glucose (PPG), glycated haemoglobin (HbA1c), serum urea, creatinine and urine albumin/creatinine ratio was performed at the initial visit and at the end of 12 weeks of treatment.

Results: Out of 84 patients screened, 74 were enrolled for the study. Of the 74 patients, 39(52.7\%) were male and 35(47.3\%) were female. The patients were divided into two groups (group A and group B) consisting 37 patients in each group. Out of 74 patients, 62 completed the study. Out of 12 patients who did not complete the study, 5 patients were lost during follow-up period and 7 patients discontinued treatment due to AEs. The mean age of the patients was 51 and 49years in the groups A and B respectively. There was no statistical difference in the baseline FPG, PPG, HbA1c, serum urea, serum creatinine, urine ACR and body weight between two groups.

Conclusions: The study shows that metformin and vildagliptin have similar effect on glycaemic control, but vildagliptin exerts better Reno protective effect and there were no reports of serious adverse events.
\end{abstract}

Keywords: Creatinine, Fasting blood glucose, Serum urea, Type 2 diabetes mellitus

\section{INTRODUCTION}

The pathophysiology of Type 2 Diabetes Mellitus (T2DM) is characterized by deficient insulin activity arising from decreased insulin secretion secondary to beta cell failure, and/or compromised insulin action in peripheral target tissues (insulin resistance). This abnormal metabolic state is exacerbated by excess hepatic glucose production and altered metabolism of proteins and lipids, which along with hyperglycemia, contribute to micro vascular and macro vascular complications. Therefore, achieving glycaemic control is a prerequisite for prevention of cardiovascular and micro vascular complications in type 2 diabetes. ${ }^{1}$ T2DM accounts for approximately $85 \%$ to $95 \%$ of diabetes patients in developed regions like the European Union (EU). Age and weight are established risk factors for T2DM. The majority of patients with T2DM are overweight or obese. Diet modification and exercise is the first line of treatment for T2DM. Pharmacologic intervention with one oral antidiabetic drug (OAD) is usually the next step in treatment. The recommended first line treatment is metformin which restrains hepatic 
glucose production and decreases peripheral insulin resistance. After 3 to 9 years of OAD monotherapy, patients typically require an additional intervention. Vildagliptin is one of a relatively new class of potential antidiabetic agents for use in the treatment of T2DM. ${ }^{2}$ Vildagliptin is an inhibitor of dipeptidyl peptidase-IV (DPP-IV), an enzyme that inactivates inter alia the incretin peptides glucagon-like peptide-1 (GLP-1) and glucosedependent insulin tropic polypeptide (GIP). ${ }^{3}$ Vildagliptin acts by increasing GLP-1 levels and thereby stimulating insulin secretion and inhibiting glucagon secretion. ${ }^{4}$ Metformin has been shown to improve insulin sensitivity in liver and muscle. ${ }^{5}$ Metformin has also been observed to increase GLP-1 levels which would be a potential for an additional synergistic action with vildagliptin. The aim of the present study was to evaluate the effect of addition of vildagliptin and fixed dose combination (FDC) of vildagliptin and metformin in T2DM patient's on insulin therapy. ${ }^{6}$

\section{METHODS}

A prospective, open-labelled, non-randomised, phase IV clinical trial was carried out over the duration of 12 weeks. The patients attending the medicine outpatient department of tertiary care teaching hospital were enrolled in the study after explaining the aim of the study. Written informed consent was obtained from each patient.

\section{Inclusion Criteria}

- $\quad$ Adults of either gender aged between 18 to 60 years.

- Newly diagnosed type-2 diabetes patients with FPG $<200 \mathrm{mg} / \mathrm{dL}, \mathrm{PPG}<300 \mathrm{mg} / \mathrm{dL}$ and HbA1clevel from 6.5 to 10 .

- Patients with normal renal function.

\section{Exclusion Criteria}

- Patients with any complication of diabetes mellitus like retinopathy, neuropathy or nephropathy etc.

- Patients with impaired liver function tests.

- Pregnant or lactating women.

- Patients taking medications that could affect blood glucose level, i.e. patients on non-selective $\beta$ blockers, diuretics and corticosteroids.

- Any contraindication to metformin/vildagliptin.

- Patients with known psychiatric illness.

Patients, who fulfilled the selection criteria, were allocated in two treatment groups (Group A and Group B) by nonrandomized manner. In both groups, age and sex matched study subjects were selected. The decision to prescribe metformin or vildagliptin was made by the treating physician according to his/her normal medical practice, and patients were enrolled in the study only after the treatment decision had been made. Group A was treated with metformin (sustained release preparation) $500 \mathrm{mg}$ once daily and group B was treated with vildagliptin 50mg once daily. Measurement of body weight, fasting blood glucose (FPG), postprandial blood glucose (PPG), HbA1c, serum urea and creatinine and urine albumin/creatinine ratio (ACR) was performed at initial visit and at the end of 12 weeks of treatment. Patients were monitored continuously throughout the study for any adverse event (AE). Adverse drug reaction form of Pharmacovigilance Programme of India (PvPI) was filled up in case of any AE. World Health Organization-Uppsala Monitoring Centre (WHO-UMC) criteria9 was used for causality assessment of AE.

\section{Statistical Analysis}

The data were analysed by SPSS version 17. $\mathrm{p}$ value of $<0.05$ was considered significant. For intragroup comparison paired $t$ test and for intergroup comparison unpaired t test were used. The data collected at 0 week will be used as the baseline against which changes during therapy will be compared. Chi-square test will be used to compare adverse effects between the groups. All statistical tests were two sided, and the results were presented as mean \pm standard deviation.

\section{RESULTS}

Out of 84 patients screened, 74 were enrolled for the study. Of the 74 patients, 39(52.7\%) were male and 35(47.3\%) were female. The patients were divided into two groups (group A and group B) consisting 37 patients in each group. Out of 74 patients, 62 completed the study. Out of 12 patients who did not complete the study, 5 patients were lost during follow-up period and 7 patients discontinued treatment due to AEs. The mean age of the patients was 51 and 49 years in the groups A and B respectively. There was no statistical difference in the baseline FPG, PPG, HbA1c, serum urea, serum creatinine, urine ACR and body weight between two groups.

\section{Hypoglycaemic Effect of Metformin and Vildagliptin}

Metformin and vildagliptin significantly reduced ( $p<0.0001)$ the levels of FPG, PPG and HbA1c at week \#12 from their respective baseline values. In both groups there was good glycaemic control $(\mathrm{HbA} 1 \mathrm{c}=6.5-8)$ at the end of the study. The reduction in blood glucose level by vildagliptin was statistically significant $(\mathrm{p}<0.001)$ as compared to metformin. But there was no significant change in the level of HbA1cbetween the groups (Table 1).

\section{Role of Metformin and Vildagliptin on Renal Function}

There was significant decrease $(\mathrm{p}<0.05)$ in serum urea level in vildagliptin group as compared to baseline value, but there was no such significant change in metformin group. In both the groups there was no significant change in serum creatinine levels at the end of 12 weeks as compared to baseline values. Vildagliptin significantly reduced urine ACR at week \#12 from baseline value $(p<0.05)$, but in metformin group there was no significant change of urine ACR after 12 weeks of therapy (Table 1). 


\section{Role of Metformin and Vildagliptin on Body Weight}

Metformin significantly reduced body weight $(\sim 3.31 \mathrm{~kg})$ at week \#12as compare to baseline value $(\mathrm{p}<0.001)$, but in vildagliptin group there was no change in the body weight (Table 1).

\section{Safety Analysis of Drug Therapy}

As per modified intention to treat (ITT), safety analysis of both metformin and vildagliptin was carried out. All patients who were receiving treatments were considered for safety analysis. Total seventeen patients developed AEs. The reported AEs were nausea, vomiting, abdominal bloating, epigastric pain, anorexia and dizziness. Ten Patients in group A and seven patients in group B reported AEs. There were no significant difference of AEs between the groups (Chi-square value $=0.6873, \mathrm{p}=0.407$ ). These AEs were mild in nature. These lasted for about 2 weeks. Four $(10.81 \%)$ patients in group A and three $(8.11 \%)$ patients in group B discontinued the study due to AEs. Causality assessment showed that they were in the "possible" category.

Table 1: Effect of Metformin and Vildagliptin on Glycaemic parameters, Kidney function and Body weight.

\begin{tabular}{|c|c|c|c|c|}
\hline \multirow{2}{*}{ Parameters } & \multicolumn{2}{|l|}{ Baseline value } & \multicolumn{2}{|c|}{ Value at the end of $12^{\text {th }}$ week } \\
\hline & Metformin group & Vildagliptin group & Metformin group & Vildagliptin group \\
\hline FPG (mg/dl) & $170.21 \pm 6.32$ & $167.56 \pm 8.57$ & $123.45 \pm 5.65$ & $116.32 \pm 10.12$ \\
\hline PPG (mg/dl) & $223.3 \pm 6.56$ & $226.12 \pm 6.59$ & $170.13 \pm 6.57$ & $158.24 \pm 12,65$ \\
\hline $\mathrm{HbA} 1 \mathrm{C}$ & $8.17 \pm 0.26$ & $8.2 \pm 0.24$ & $6.85 \pm 0.2$ & $6.86 \pm 0.14$ \\
\hline Serum urea $(\mathrm{mg} / \mathrm{dl})$ & $31.35 \pm 5.45$ & $32.65 \pm 8.62$ & $30.13 \pm 5.02$ & $26 \pm 5.64$ \\
\hline $\begin{array}{l}\text { Serum creatinine } \\
(\mathrm{mg} / \mathrm{dl})\end{array}$ & $0.65 \pm 0.19$ & $0.8 \pm 0.2$ & $0.62 \pm 0.07$ & $0.65 \pm 0.05$ \\
\hline Urine ACR (mg/g) & $17.67 \pm 3.15$ & $23 \pm 5.70$ & $17.43 \pm 1.56$ & $16.54 \pm 3.23$ \\
\hline Body Weight (Kg) & $67.12 \pm 4.56$ & $65.12 \pm 4.62$ & $64 \pm 3.56$ & $65.12 \pm 4.23$ \\
\hline
\end{tabular}

\section{DISCUSSION}

In this study, metformin significantly reduced the levels of FPG, PPG and HbA1c after 12 weeks of therapy. These findings are similar to that of ADAM CR et al.7 Metformin improves hyperglycaemia primarily by suppression of hepatic gluconeogenesis. ${ }^{11}$ In addition to suppressing hepatic gluconeogenesis, metformin increases insulin sensitivity, enhances peripheral glucose uptake and increases fatty acidoxidation. ${ }^{12}$ Vildagliptin also significantly reduced the levels of FPG, PPG and HbA1c after 12 weeks of therapy. It acts by increasing the levels of GLP-1 and GIP by inhibiting DPP IV enzyme which is responsible for degradation of GLP-1 and GIP. ${ }^{13}$ Vildagliptin caused more reduction of FPG and PPG but similar reduction of $\mathrm{HbA1c}$ after 12 weeks of therapy as compared to that of metformin. ${ }^{8}$ Both metformin and vildagliptin protected renal function. Vildagliptin in addition improves kidney function as there was improvement in serum urea and urinary albumin excretion. Liu et al, reported that vildagliptin attenuates kidney injury in streptozotocin-induced diabetic rats. In this same model, vildagliptin also significantly decreased proteinuria, albuminuria and urinary ACR..$^{9,10}$ Metformin significantly reduced body weight $(\sim 3.31 \mathrm{~kg})$ which is comparable with the finding of Jack A. Yanovski et al, But Overall weight neutrality has been seen with vildagliptin which is similar with findings of other studies. The overall weight neutrality seen with vildagliptin appears to be a class effect because the DPP-4 inhibitors, saxagliptin and sitagliptin have also been shown to produce improvements in glycaemic control, both as monotherapy and as add-on therapy to other oral agents, without significant change in body weight in most clinical trials. ${ }^{11}$ Safety analysis showed that there were no serious AEs, although $10.81 \%$ patients in group $\mathrm{A}$ and $8.11 \%$ patients in group $\mathrm{B}$ discontinued treatment due to AEs. ${ }^{12}$

\section{CONCLUSION}

The study shows that metformin and vildagliptin have similar effect on glycaemic control, but vildagliptin exerts better Reno protective effect and there were no reports of serious adverse events.

Funding: No funding sources Conflict of interest: None declared

Ethical approval: The study was approved by the Institutional Ethics Committee

\section{REFERENCES}

1. Robinson AC, Burke J, Robinson S, Johnston DG, Elkeles RS. The effects of metformin on glycemic control and serum lipids in insulin-treated NIDDM patients with suboptimal metabolic control. Diabetes Care. 1998 May 1;21(5):701-5.

2. Dmitri K, McFarlane Samy I, Sowers James R. Metformin:an update. Ann Intern Med. 2002 Jul 2;137(1):25-33. 
3. Collier CA, Bruce CR, Smith AC, Lopaschuk G, Dyck DJ. Metformin counters the insulin-induced suppression of fatty acid oxidation and stimulation of triacylglycerol storage in rodent skeletal muscle. Am J Physiol Endocrinol Metab. 2006 Jul;291(1):E182-9.

4. Sebokova E, Christ AD, Boehringer M, Mizrahi J. Dipeptidyl peptidase IV inhibitors: the next generation of new promising therapies for the management of type 2 diabetes. Current Topics in Med Chem. 2007 Mar 1;7(6):547-55.

5. Liu WJ, Xie SH, Liu YN, Kim W, Jin HY, Park SK, et al. Dipeptidyl peptidase IV inhibitor attenuates kidney injury in streptozotocin-induced diabetic rats. J Pharmacol Exp Ther. 2012 Feb 1;340(2):248-55.

6. Yanovski JA, Krakoff J, Salaita CG, McDuffie JR, Kozlosky M, Sebring NG, et al. Effects of metformin on body weight and body composition in obese insulin-resistant children. Diabetes. 2011 Feb $1 ; 60(2): 477-85$.

7. Nathan DM, Buse JB, Davidson MB, Ferrannini E, Holman RR, Sherwin R, et al. Medical management of hyperglycemia in type 2 diabetes: aconsensus algorithm for the initiation and adjustment of therapy: aconsensus statement of the American Diabetes.

8. Association and the European Association for the Study of Diabetes. Diabetes Care. 2009 Jan 1;32(1):193-203.

9. Pi-Sunyer FX, Schweizer A, Mills D, Dejager S. Efficacy and tolerability of vildagliptin monotherapy in drug-naïve patients with type 2 diabetes. Diabetes Res Clin Pract. 2007 Apr 1;76(1):132-8.

10. Dejager S, Razac S, Foley JE, Schweizer A. Vildagliptin in drug-naïve patients with type 2 diabetes: a24-week, double-blind, randomized, placebo-controlled, multiple-dose study. Horm Metab Res. 2007 Mar;39(03):218-23.

11. Garber AJ, Foley JE, Banerji MA, Ebeling P, Gudbjörnsdottir S, Camisasca RP, Couturier A, Baron MA. Effects of vildagliptin on glucose control in patients with type 2 diabetes inadequately controlled with a sulphonylurea. Diabetes Obes Metab. 2008 Nov;10(11):1047-56.

12. Rosenstock J, Sankoh S, List JF. Glucose-lowering activity of the dipeptidyl peptidase-4 inhibitor saxagliptin in drug-naive patients with type 2 diabetes. Diabetes Obes Metab. 2008 May;10(5):376-86.

13. Aschner P, Kipnes MS, Lunceford JK, Sanchez M, Mickel C, Williams-Herman DE. Effect of the dipeptidyl peptidase-4 inhibitor sitagliptin as monotherapy on glycemic control in patients with type 2 diabetes. Diabetes Care. 2006 Dec 1;29(12):2632-7.

Cite this article as: Lavakumar S, Jesurun JRS. A prospective open labelled study of effect of vildagliptin with metformin in patients with type 2 diabetes mellitus. Int J Basic Clin Pharmacol 2019;8:1880-3. 